CBIE-LACLO 2015

Anais dos Workshops do IV Congresso Brasileiro de Informática na Educação (CBIE 2015)

\title{
GeoTouch: Sistema de Geometria Interativa para Dispositivos Móveis
}

\author{
Helena M. Reis, Seiji Isotani \\ Universidade de São Paulo - Instituto de Ciências Matemáticas e Computação (ICMC) \\ Caixa Postal 668 - CEP 13560-970 - São Carlos - SP - Brazil \\ \{helenamcd, sisotani\}@icmc.usp.br
}

\begin{abstract}
Resumo: Os sistemas de Geometria Interativa (SGI) foram desenvolvidos com o objetivo de possibilitar aos estudantes explorarem a geometria por meio do computador. Tradicionalmente nos SGI, o usuário-aprendiz constrói um objeto geométrico usando o ícone a ele associado disponível na interface do sistema. Se de um lado a literatura indica que este tipo de sistema pode facilitar o aprendizado de Geometria, de outro existem estudos sugerindo que a redução de icones na interface pode ser benéfica para os usuários iniciantes, diminuindo a dificuldade de se localizar o icone desejado em meio a vários outros. Neste contexto, o presente artigo propõe a redução de ícones nas interfaces dos SGI por meio da definição de uma família de gestos naturais que podem ser utilizados para construção e manipulação de objetos geométricos. Para utilizar e validar os gestos propostos desenvolveu-se um SGI utilizando Interface Gestual para uso em dispositivos móveis chamado Geotouch.
\end{abstract}

\section{Cenário de Uso}

Os sistemas de Geometria Interativa (GI) possibilitam o aprendiz a explorar construções geométricas do tipo régua-e-compasso com o uso computador (Kortenkamp et al., 2004). No início dos anos 2000 vários desses sistemas apresentavam interfaces com um grande número ícones, o que poderia dificultar o trabalho, principalmente de um usuário iniciante. Esta dificuldade foi constatada em alguns estudos que analisaram o número de ícones disponíveis na interface de sistemas de GI, como em Schimpf e Spannagel (2011) e Reis et al. (2012). Uma possível proposta para reduzir a dificuldade provocada pelo número de ícones disponíveis é o emprego de gestos, principalmente em dispositivos com telas sensíveis ao toque (Kortenkamp e Dohrmann, 2010).

Apesar de existir sistemas de GI que utilizam a interação por meio de gestos em dispositivos móveis (e.g. Geogeobra, Skecthometry e Geometry Pad), estes sistemas não consideram os conceitos matemáticos de cada objeto construído. Por exemplo, se uma circunferência for desenhada com o gesto de um círculo, o aluno não vai associar que toda circunferência possui um raio de tamanho $r$ e que todos os pontos desta circunferência estão à distância $r$ do seu centro (ou seja, são equidistantes do centro), gerando problemas conceituais que podem afetar a aprendizagem. Para amenizar este problema, é importante a significância de cada gesto realizado, ou seja, que os conceitos matemáticos subjacentes à construção sejam utilizados para definir um gesto. Além dos benefícios pedagógicos que esta abordagem oferece, também existem vantagens relacionadas ao desenvolvimento de uma interface de GI, pois o estudante pode aplicar 
o conhecimento prévio sobre geometria para interagir com o software e realizar suas ações.

Neste contexto, o foco deste artigo é apresentar o desenvolvimento de um novo sistema de GI, GeoTouch, para dispositivos móveis, cuja forma de interação está baseada em gestos/toques. Deste modo, o aprendiz pode realizar alguma construção sem a necessidade de encontrar e ativar um ícone relacionado. Além disso, no GeoTouch os gestos foram baseados nos conceitos geométricos subjacentes, sendo o modelo de gestos uma das contribuições do protótipo.

\section{Desenvolvimento}

A partir dos resultados apresentados em Vitale et al. (2014), realizou-se entrevistas com especialistas da área de matemática, a fim de investigar quais atividades são realizadas dentro do ambiente de ensino para apoiar a aprendizagem de geometria utilizando um software de GI. Nesta fase, quatro professoras com doutorado em Educação Matemática com mais de 20 anos de atuação profissional foram entrevistadas individualmente durante o período de aproximadamente uma hora.

Os dados das entrevistas foram transcritos e tabulados para auxiliar na definição tanto da família de gestos para construção de objetos geométricos quanto dos requisitos de um sistema de GI para dispositivos móveis. Em particular, as entrevistas apontaram que o aprendizado dos conceitos geométricos pode ser dificultado se os gestos criados não tiverem relação com as propriedades geométricas dos objetos corroborando com as ponderações feitas por Vitale et al. (2014).

Com base nas funcionalidades e requisitos identificados nas entrevistas, foram elaborados o caso de uso, diagrama de classes e o fluxograma das ações ao tocar na tela, que podem ser visualizados pelo endereço http://goo.gl/5Oacf5. Além disso, foi elaborado um dicionário de gestos para realizar a construção de objetos geométricos e interagir com o sistema. $\mathrm{O}$ dicionário de gestos é composto pela descrição textual do objeto, sua relação com os conceitos geométricos (caso exista) e um conjunto de figuras ilustrativas que apresentam como realizar os gestos em uma interface baseada em tela multitoque. Inicialmente foram gerados múltiplos gestos para atender uma mesma funcionalidade ou requisito e, após múltiplas prototipações e interações com usuários, foram definidas três categorias de gestos para sistematizar a forma como interagir com o sistema e permitir um alto grau de flexibilidade e extensibilidade. Estas categorias são apresentas a seguir:

- Gestos do Núcleo (ou Gesto Base): gestos principais do software e que são a base para a definição dos gestos de outras categorias;

- Gestos de Navegação: gestos para a navegação no software, os quais agrupam gestos relacionados à manipulação/edição de objetos geométricos;

- Gestos Básicos de Construção: gestos que combinam gestos de núcleo e são usados para a construção dos objetos geométricos simples.

Para maiores detalhes, o dicionário de gestos pode ser visualizado no endereço http://goo.gl/5Oacf5. Utilizando o dicionário de gestos desenvolvido neste trabalho, foi proposta a criação de um software de Geometria Interativa para dispositivos móveis com tela multitouch, chamado de GeoTouch. 
Após o seu desenvolvimento, foi realizada uma prova de conceitos com o objetivo de comparar a forma de interação proposta para construção de objetivos geométricos por meio de gestos com outros softwares de GI. Além disso, um outro objetivo foi também verificar problemas de usabilidade com o software de GI desenvolvido, GeoTouch. A técnica selecionada para a prova de conceitos foi a análise de usabilidade utilizando a avaliação heurística definida por Nielsen (1993). O teste foi realizado em 4 etapas: (i) leitura das heurísticas, (ii) execução de tarefas, (iii) solução de problemas de geometria e (iv) preenchimento do formulário sobre os problemas encontrados de acordo com suas heurísticas. Os formulários utilizados e os resultados podem ser acessados pelo endereço http://goo.gl/5Oacf5

\section{Apresentação do software}

A versão inicial foi desenvolvida na plataforma Android versão 4.0 e pode ser executada em dispositivos móveis, como tablets de 7 e 10 polegadas ou smartphones. Atualmente, o GeoTouch está disponível gratuitamente na loja virtual de aplicativos da Google (o Google Play) e pode ser acessado por meio do link: http://www.icmc.usp.br/e/feb45.

A interação com o software pode ser realizada por meio de um ou mais dedos, e as construções básicas dos objetos geométricos são feitas por meio dos gestos definidos na seção anterior. Isso permitiu uma sensível redução do número de ícones em tela. A Figura 1.a mostra a tela de apresentação do aplicativo, que foi nomeado GeoTouch. A Figura 1.b mostra a tela inicial do protótipo, na qual é possível identificar que o lado direito é a área de desenho, em que os usuários podem construir os objetos geométricos, e o lado esquerdo, fica localizado o menu. Este menu é acessado por meio de um ícone localizado na parte superior esquerda e foi desenvolvido de modo que o usuário consiga escondê-lo quando desejar trabalhar na área de desenho e mostrá-lo quando precisar acessar alguma funcionalidade do menu.

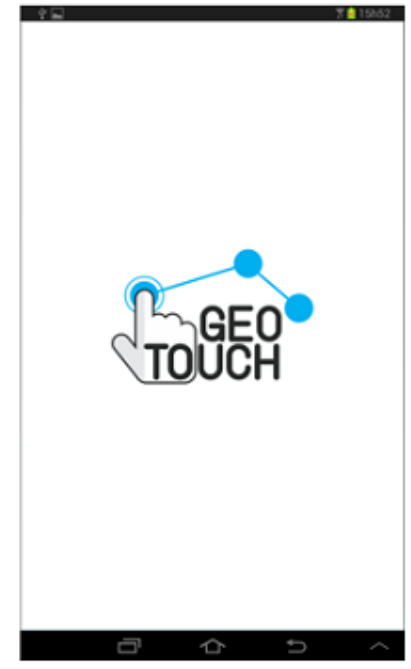

(a)

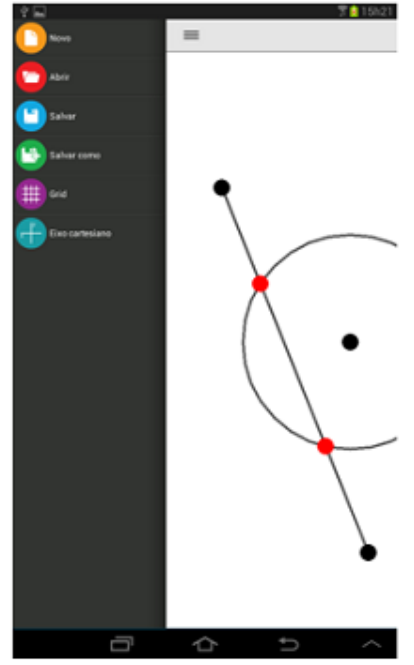

(b)

Figura 1: Tela de apresentação e inicial do software GeoTouch.

O Geotouch possui todas as funcionalidades que envolvem construções básicas de objetos geométricos para o ensino da geometria euclidiana. Além da construção destes objetos, também é possível a sua manipulação (e.g. movimentação de um objeto) 
e edição (e.g. aumentar o tamanho de uma reta ou circunferência). Outras funcionalidades elementares também foram desenvolvidas (e.g. Novo, Abrir, Salvar, Exibir Eixo Cartesiano, Medir Distância). Um vídeo ilustrativo do uso do GeoTouch está disponível a partir do link: http://www.icmc.usp.br/e/185e3

\section{Conclusões}

Acredita-se que o GeoTouch possa se tornar uma ferramenta de referência que pode ser utilizada para auxiliar alunos e professores no processo de ensino e aprendizagem da geometria utilizando dispositivos móveis. Este auxílio se dá não apenas pelos recursos do software, mas também pelo fato de todo o desenvolvimento estar fortemente relacionando aos conceitos geométricos subjacentes à construção dos objetos geométricos, a fim de facilitar o aprendizado da interface e do conteúdo.

Contudo, vale ressaltar que embora a realização da avaliação heurística permitir que os gestos e a interface propostas fossem avaliados no aspecto de usabilidade, observa-se ainda há a necessidade de uma avaliação com alunos e professores em ambientes reais de ensino-aprendizagem, com atividades que os estudantes executam diariamente em sala de aula. Assim, como trabalhos futuros pretende-se realizar experimentos em ambientes reais, com alunos e professores, para validar o ganho pedagógico ao utilizar o Geotouch. Além disso, em futuras versões deste sistema serão desenvolvidas as funcionalidades de criação de ponto sobre objetos, cálculo de ângulos, inclusão de rótulos, compartilhamento e colaboração síncrona de construções geométricas e avalição automática de exercícios.

\section{References}

Kortenkamp, U.; Dohrmann, C. (2010). User interface design for dynamic geometry software. Acta Didactica Napocensia, 3(2). Available at http://dppd.ubbcluj.ro/adn/article_3_2_6.pdf

Kortenkamp, U.; and Richter-Gebert, J. (2004) Using automatic theorem proving to improve the usability of geometry software. Procedings of the Mathematical User-Interfaces Workshop. Available at: http://kortenkamps.net/papers/2004/ATP-UI-article.pdf

Laborde, C. (2007). The role and uses of technologies in mathematics classrooms: Between challenge and modus vivendi. Canadian Journal of Science, Mathematics and Technology Education, 7(1), 68-92.

Nielsen, J. (1993). Usability Engineering. San Francisco, CA.

Reis, H. M.; Borges; S.; Isotani, S. (2013). Interação em interfaces de softwares de geometria interativa: Um mapeamento sistemático. In: Simpósio Brasileiro de Informática na Educação (SBIE), páginas 1-10.

Santos, E.T.; Lourenzoni, L.; Oliveira, A. L. L. (2005) RISKO: desenhando com instrumentos virtuais. Simpósio Nacional de Geometria Descritiva e Desenho Técnico/V International Conference on Graphics Engineering for Arts and Design. Available at: http://www.gente.eti.br/lematec/CDS/GRAPHICA05/artigos/santoslourenzonideoliveira.p df

Schimpf, F.; Spannagel, C. (2011). Reducing The Graphical User Interface Of A Dynamic Geometry System. ZDM, 43(3), 389-397.

Vitale, J. M.; Swart, M. I.; Black, J. B. (2014). Integrating Intuitive And Novel Grounded Concepts In A Dynamic Geometry Learning Environment. Computers and Education, 72(March),231-248. 